\title{
Intratumoral radiofrequency hyperthermia-enhanced direct chemotherapy of pancreatic cancer
}

\author{
Zhibin Bai ${ }^{1,2 *}$, Yaoping Shi ${ }^{1,3 *}$, Jianfeng Wang ${ }^{1}$, Longhua Qiu ${ }^{1}$, Eric J. Monroe ${ }^{1}$, \\ Gaojun Teng ${ }^{2}$, Feng Zhang ${ }^{1, * *}$ and Xiaoming Yang ${ }^{1, * *}$ \\ ${ }^{1}$ Image-Guided Biomolecular Intervention Research, Section of Interventional Radiology, Department of Radiology, University \\ of Washington School of Medicine, Seattle, WA, USA \\ 2 Department of Radiology, Zhongda Hospital, Southeastern University, Nanjing, China \\ ${ }^{3}$ Department of Interventional Oncology, Renji Hospital, School of Medicine, Shanghai Jiaotong University, Shanghai, China \\ * These authors made the equal contributions as the co-first author for this study \\ ** These authors made the equal contributions for this study \\ Correspondence to: Xiaoming Yang, email: xmyang@uw.edu
}

Keywords: radiofrequency, hyperthermia, chemotherapy, pancreatic cancer, local

Received: April 06, $2016 \quad$ Accepted: September 20, $2016 \quad$ Published: September 27, 2016

\section{ABSTRACT}

Purpose: To investigate the technical feasibility of using ultrasound-guided intratumoral radiofrequency hyperthermia (RFH) to enhance local chemotherapy of rat orthotopic pancreatic cancers.

Materials and Methods: Orthotopic pancreatic cancer masses were established by inoculating luciferase/mCherry labeled-pancreatic cancer cells into the pancreatic tails of Lewis model rats via a laparotomy approach. Twenty-four rats with pancreatic cancer and 24 mice with subcutaneous pancreatic cancer xenografts in four study groups ( $n=6 /$ group) received various treatments: $i$ ) combination therapy of intratumoral MR imaging-heating-guidewire-mediated $\mathrm{RFH}\left(42^{\circ} \mathrm{C}\right)$ plus local chemotherapy (gemcitabine); ii) intratumoral chemotherapy alone; iii) RFH alone; and (iv)phosphate-buffered saline (PBS). Transcutaneous ultrasound imaging was used to guide the treatment and subsequently follow changes in tumor sizes. Bioluminescence optical imaging was performed to follow photon signal changes. Sonographic and optical findings were correlated with histology at 14 days.

Results: Optical imaging demonstrated a significantly decreased bioluminescence signal in mice with combination therapy group, compared with the other control groups (0.51 \pm 0.18 VS $1.6 \pm 0.4$ VS $3.18 \pm 0.9$ VS $3.5 \pm 0.96, p<0.05)$. Ultrasound imaging showed the smallest tumor volumes of both mice and rat group with the combination therapy, compared with other control groups $(0.62 \pm 0.16$ VS $1.25 \pm 0.19$ VS $2.28 \pm 0.25$ VS $2.64 \pm 0.26, p<0.05)$ and $(0.75 \pm 0.18$ VS $1.31 \pm 0.30$ VS $1.61 \pm 0.28$ VS $1.72 \pm 0.28, p<0.05)$. Both imaging findings were confirmed by histologic correlation.

Conclusion Intratumoral RFH can augment the chemotherapeutic effect in an orthotopic pancreatic cancer model.

\section{INTRODUCTION}

Pancreatic cancer is one of the most lethal abdominal malignancies and is the fourth leading cause of cancer-related death in Western nations. At the time of diagnosis, more than $80 \%$ of patients present with either an advanced unresectable tumor or metastatic disease [1, 2]. In cases of unresectable disease, the median survival is around 6 months [3-6]. Therefore, it is essential to develop novel and effective therapies for patients with unresectable pancreatic cancer [4-6].

Gemcitabine-based chemotherapy has been accepted as the first line treatment for patients with unresectable and locally advanced pancreatic cancer, but offers only a limited survival benefit of 3 months [7-9]. The unsatisfactory therapeutic effect of systemic chemotherapy 
is associated with the difficulty of delivering sufficient drugs to highly desmoplastic pancreatic cancer tissues [8]. Radiofrequency (RF)-mediated ablative thermal energy has been increasingly used to treat solid parenchymal tumors. RF ablation of unresectable pancreatic tumors has been reported [3, 7, 9-11], but carries a high risk of pancreatitis and severe thermal injuries to critical structures adjacent to the pancreatic tumors, such as duodenum, portal vein or common bile duct [3, 12-14]. Some serious complications require operative intervention and therefore surgery despite the presence of unresectable tumor [3].

Recent studies have confirmed that subablative hyperthermia around $42^{\circ} \mathrm{C}$ can significantly improve chemotherapeutic effect on different cancers via increasing the sensitivity of cancer cells to chemotherapeutic drugs and reversing chemo-resistance [1, 15, 16]. However, in clinical practice, such subablative hyperthermia for treating malignancies has been generated by either whole body hyperthermia or external hyperthermia around the body $[1,17,18]$. In the last decade, we have developed a radiofrequency heating guidewire, which functions as an intraluminal thermal energy source for locally enhancing gene/chemotherapies $[16,19,20]$. The purpose of this study was to investigate the technical feasibility of using ultrasound-guided intratumoral radiofrequency hyperthermia (RFH) to enhance local chemotherapy of rat orthotopic pancreatic cancers.

\section{MATERIALS AND METHODS}

\section{Study design}

We divided the study into three phases: (1) in-vitro experiments to establish "proof-of-principle" of the new concept, RFH-enhanced chemotherapy of pancreatic cancer, using rat pancreatic cancer cells (DSL-6A/C1); (2) in-vivo confirmation of the new concept on mice models with molecular imaging-detectable subcutaneous pancreatic cancer xenografts; and (3) preclinical feasibility validation of the new technique using a rat models with orthotopic pancreatic cancers.

\section{In vitro experiments}

\section{Cell culture and RFH-enhanced chemotherapy}

Rat pancreatic cancer cells (DSL-6A/C1) were transfected with luciferase (Luc)/red fluorescence protein (RFP) /lentivirus gene to create Luc/RFP-positive cells according to the protocol provided by the manufacturer (GeneCopoeia Inc., Rockville, MD). Luc/RFP-positive cells were sorted out using fluorescence-activated cell sorting technique (Aria II, Becton Dickinson, Franklin
Lakes, NJ). Cells were then seeded in four-chamber cell culture slides (Nalge Nunc International, Rochester, NY) and maintained in Waymouth's MB 752/1 medium (Gibco, Grand Island, NY) supplemented with 10\% fetal bovine serum (FBS) (Gibco). RF hyperthermia was performed as described in the literature [20]. Cells in different groups were treated by (a) Gemcitabine $(5.0 \mu \mathrm{M})$ plus 30 -min $\mathrm{RFH}$ at approximately $42^{\circ} \mathrm{C}$; (b) Gemcitabine alone; (c) 30-min RFH alone; and (d) no treatment to serve as a control. We used the 50-percentage inhibitory concentration (IC50) dose of gemcitabine for cell treatment, which was decided by CellTiter 96 Aqueous One Solution Cell Proliferation-assay (Promega Corporation, Madison, WI).

\section{Cell viability assay}

Cells proliferation was evaluated by CellTiter 96 Aqueous One Solution Cell Proliferation-assay 48 hours after treatments. Relative cell proliferations of different cell groups were evaluated using the equation of $\mathrm{A}_{\text {treated }}{ }^{-}$ $\mathrm{A}_{\text {blank }} / \mathrm{A}_{\text {control }}-\mathrm{A}_{\text {blank }}$, where $\mathrm{A}$ is absorbance. Cells on slides were subsequently washed twice with phosphatebuffered saline (PBS), fixed in 4\% paraformaldehyde, counterstained with 4',6-diamidino-2-phenylindole (DAPI, Vector Laboratories, Burlingame, CA), and then imaged with a confocal microscopy. All experiments for each of cell groups were repeated six times.

\section{In vivo experiments}

The animal protocol was approved by our Institutional Animal Care and Use Committee. The animals were anesthetized with 1\%-3\% isoflurane (Piramal Healthcare, Andhra Pradesh, India) in 100\% oxygen.

\section{Mouse model for proof of principle}

Mouse models with pancreatic cancer xenografts were created on $24 \mathrm{nu} /$ nu mice aged 4-6 weeks (Charles River Laboratories, Wilmington, MA) by subcutaneously inoculating $5 \times 10^{6}-1 \times 10^{7}$ Luc/RFP-positive DSL-6A/C1 cells in $100 \mu \mathrm{l}$ Matrigel into the left back of each mouse. Once the size of tumor reached $5-10 \mathrm{~mm}$ in diameter, we began the experimental procedures.

When the tumors grew to around 5-10 $\mathrm{mm}$ in diameter, six mice in each of four groups were treated by (a) intratumoral injections of gemcitabine $(20-\mathrm{mg} / \mathrm{kg}$ for mice) in $100 \mu \mathrm{L} \mathrm{PBS}$, followed by RFH at approximately $42^{\circ} \mathrm{C}$ for 30 mins; (b) intratumoral injection of gemcitabine alone; (c) 30-min RFH alone; and (d) injection of $100 \mu \mathrm{L}$ PBS to serve as a control. RF hyperthermia was performed as described in the literature [16].

\section{Pre-clinical validation of the new technique feasibility using rat models}

Rat models with orthotopic pancreatic cancer xenografts were created on Lewis rats weighted 150-200g 
(Harlan Laboratories, Livermore, CA). A total of $5 \times 10^{6}$ $1 \times 10^{7} \mathrm{DSL}-6 \mathrm{~A} / \mathrm{C} 1$ cells were first subcutaneously injected into right flanks of 6 donor Lewis rats. The subcutaneous tumors were excised under aseptic conditions once they grew to $10-15 \mathrm{~mm}$ in the largest diameter. The tumors were harvested and minced by a scalpel into small fragments of $1 \mathrm{~mm}^{3}$ in size.

For each of the 24 recipient Lewis rats, the abdomen was opened through a median incision and the spleen pancreas and pancreatic tail were gently exposed. A tumor bed was prepared in the pancreatic parenchyma with a microscissor (RS-5610 VANNAS; Roboz, Rockville, MD). Five tumor fragments were placed into pancreatic tissue bed, completely wrapping the tumor tissue by pancreatic parenchyma. The pancreas was relocated into the abdominal cavity and the median incision was closed in two layers with 5-0 absorbable sutures.

When the orthotopic pancreatic tumors had grown to $5-10 \mathrm{~mm}$ in diameter, six rats in each of four groups were treated by (a) intratumoral injections of gemcitabine (100-mg/kg for rats) in $100 \mu \mathrm{L} \mathrm{PBS,} \mathrm{followed} \mathrm{by} \mathrm{RFH} \mathrm{at}$ approximately $42^{\circ} \mathrm{C}$ for 30 mins; (b) intratumoral injection of gemcitabine alone; (c) 30-min RFH alone; and (d) injection of $100 \mu \mathrm{L}$ PBS to serve as a control. Gemcitabine in $100 \mu \mathrm{L}$ PBS was directly injected into the tumor mass through the percutaneous puncture approach under ultrasound guidance. Immediately after the drug delivery, RF hyperthermia was delivered by inserting a 0.022 -inch MR imaging heating guidewire into tumor mass via the gemcitabine injection needle, with its heating spot located at the center of tumor mass. A fiberoptic temperature probe was placed in the tumor margin parallel to MR imaging heating guidewire to monitor the temperature under ultrasound guidance. The temperature was kept at around $42^{\circ} \mathrm{C}$ for 30 minutes by adjusting the RF output power at 10 watts.

Following tumor growth with bioluminescent (BL) optical imaging and ultrasound imaging

Optical imaging was conducted on a Bruker InVivo Xtreme Imaging Systems (Bruker Corp., Billerica, MA). For mice, each animal was imaged at day 0 before treatment and at days 7 and 14 after the treatment. Animals were anesthetized with 1\%-3\% isofluorane in $100 \%$ oxygen. Bioluminescent images were acquired twenty minutes after intraperitoneal injection of Pierce D-Luciferin $(150 \mathrm{mg} / \mathrm{Kg}) \quad$ (ThermoFisher Scientific, Pittsburgh, PA). Signal intensity was quantified using the Bruker MI software. Relative signal intensity (RSI) was calculated by using the equation: $\mathrm{RSI}=\mathrm{SI}_{\mathrm{Dn}} / \mathrm{SI} \mathrm{D}_{\mathrm{D} 0}$, where SI is signal intensity, Dn represents days after treatment, and D0 is the day before treatment.

Ultrasound imaging was performed to follow the tumor growth (Sonosite Inc, Bothel, WA) at day 0 before treatment and at days 7 and 14 after the treatment for both mice and rats. The axial (X) and longitudinal (Y) diameters of tumors, as well as tumor depths (Z) were measured on the ultrasound images. The volume of each tumor mass was calculated according to equation of volume $=X^{*} Y^{*} Z^{*} \pi / 6$. Data was expressed as relative tumor volume (RTV) by using the following equation: $\mathrm{RTV}=\mathrm{V}_{\mathrm{Dn}} / \mathrm{V}_{\mathrm{D} 0}$, where $\mathrm{V}$ is tumor volume, Dn represents days after treatment, and D0 is the day before treatment.

\section{Histologic correlation/confirmation}

Tumors were harvested 14 days after treatments and cryosectioned at $8-\mu \mathrm{m}$ slices for apoptosis staining. Level of apoptosis was determined with a terminal deoxynucleotidyl transferase dUTP nick end labeling assay (TUNEL) using TACS XL Blue Label kit (Trivegen, Gaithersburg, MD). On one slide, six fields were randomly photographed using an Olympus DP72 digital camera. Apoptosis results were analyzed as the apoptotic index, defined as the number of apoptotic cells / total number of cells $\times 100 \%$.

\section{Statistical analysis}

Statistical software SPSS 19.0 (SPSS, Chicago, Ill) was used for all data analyses. A non-parametric Mann-Whitney $U$ test was used to compare (i) relative proliferation rates among different cell groups; (ii) relative optical signal intensities as well as (iii) relative tumor volumes at different time points among various animal groups. $\mathrm{P}$ value of less than 0.05 was considered significant.

\section{RESULTS}

\section{In vitro confirmation: RFH-enhanced chemotherapeutic effect on pancreatic cancer cells}

Confocal microscopy of cells in each treated group showed that much less cells survived after the treatment in combination therapy than three other groups (Figure 1a), which was consistent with the results of proliferation assay. CellTiter 96 Aqueous One Solution Cell Proliferation-assay demonstrated the lowest cell viability of Chemotherapy plus RFH, compared to those of groups with chemotherapy-only, RFH-only, and PBS $(32.71 \pm 1.34 \%$ VS $50.22 \pm 3.96 \%$ VS $99.23 \pm 6.09 \% \quad V S$ $100 \%, p<0.05$ ) (Figure 1b).

\section{In vivo confirmation: RFH-enhanced chemotherapy on mouse subcutaneous pancreatic cancers}

All mice survived the procedures without any major complications. Optical imaging showed a significant decrease of relative photon signal intensities for the combination therapy group as compared to those 

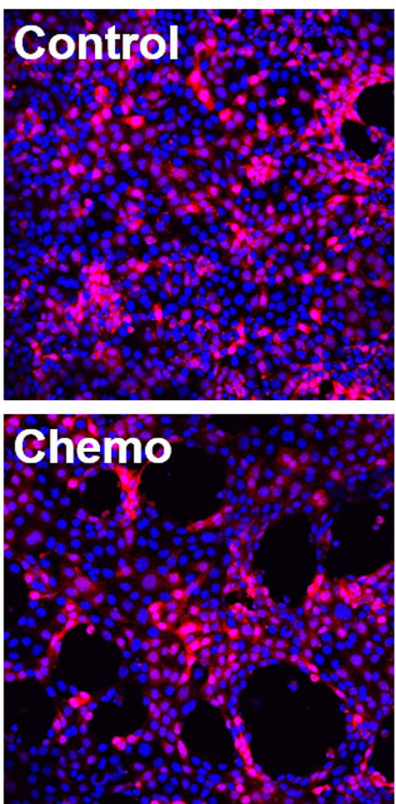

a

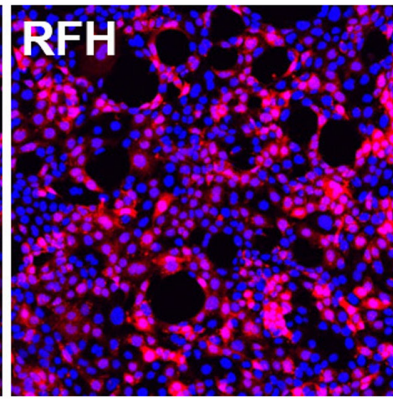

\section{Chemo+RFH}

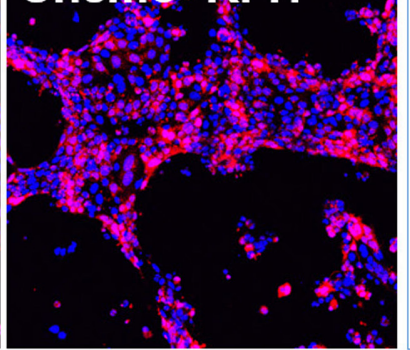

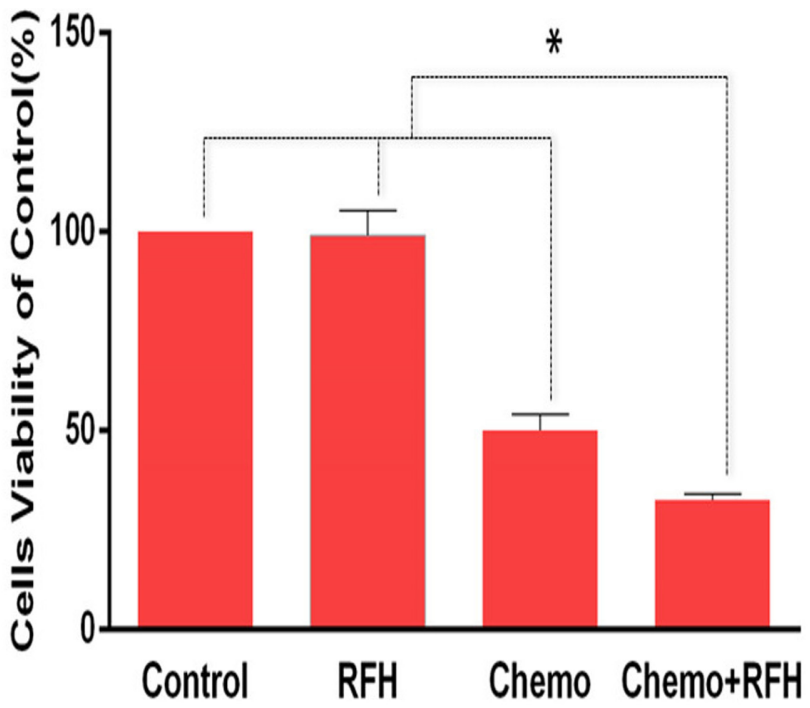

Figure 1: In vitro experiments using Luciferase-labelled pancreatic cancer cells. a. Confocal microscopy shows the lowest numbers of survival cells in the combination treatment (Chemo+RFH). b. MTS assay demonstrating the lowest cell viability in the group receiving combination treatment $(\mathrm{Chemo}+\mathrm{RFH})$, compared with those of control groups $(*=p<0.05)$.
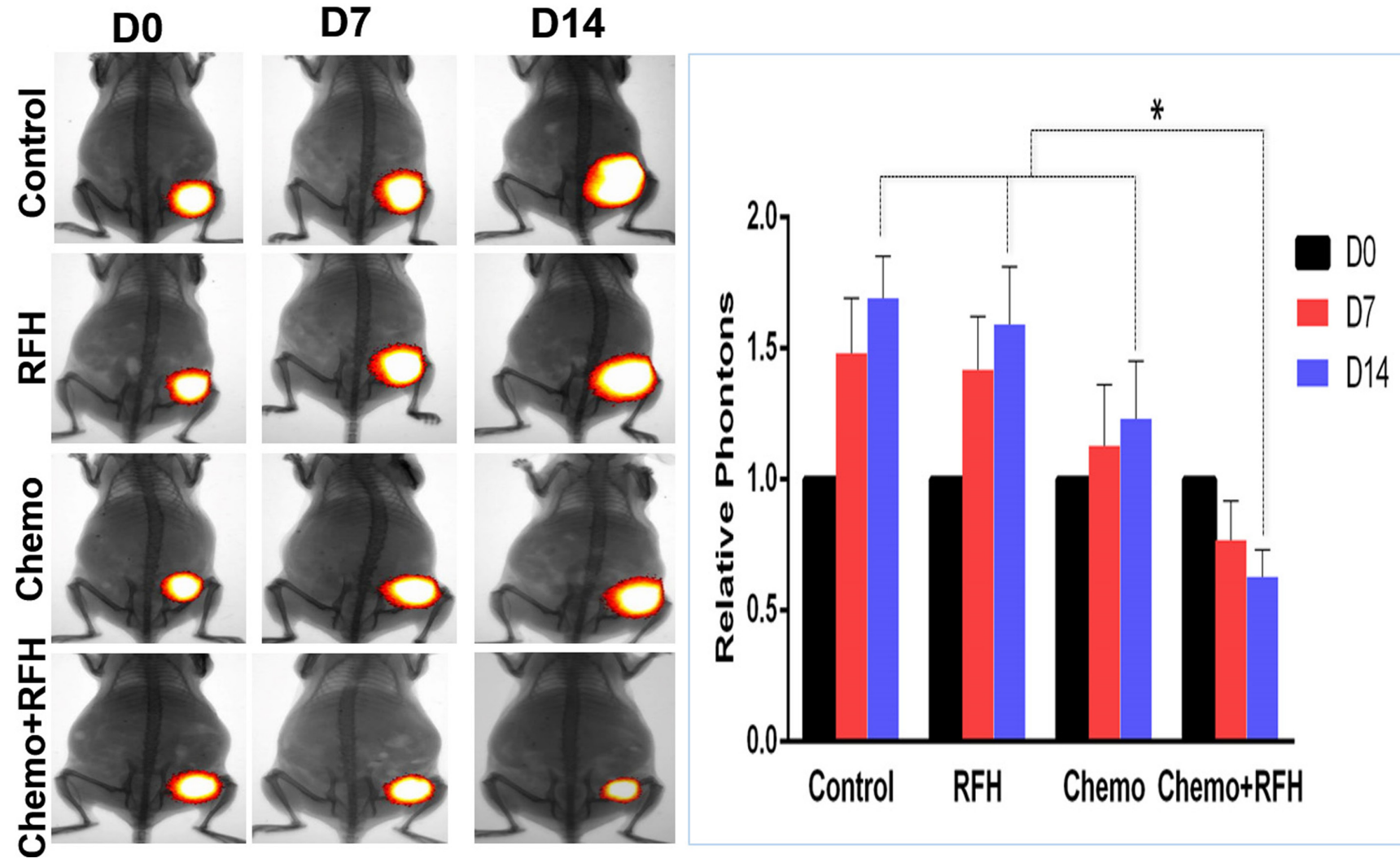

Figure 2: In vivo experiments on mouse models with subcutaneous pancreatic cancers. (Left) Optical/x-ray imaging is used to follow up the tumor growth at days 0,7 and 14 after treatments, showing significantly decreased florescent signals (yellow-red color) in the group with combination therapy $(\mathrm{Chemo}+\mathrm{RFH})$, compared to those of other three control groups (Right, $* p<0.05)$. 
of chemotherapy-only group, RFH-only group, and control group $(0.63 \pm 0.10 \mathrm{VS} 1.23 \pm 0.22 \mathrm{VS} 1.59 \pm 0.22 \mathrm{VS}$ $1.69 \pm 0.16, p<0.05$ ) (Figure 2). Ultrasound imaging of tumors showed the smallest relative tumor volumes in combination therapy group compared with control groups $(0.62 \pm 0.06$ VS $1.20 \pm 0.16$ VS $1.56 \pm 0.19$ VS $1.65 \pm 0.18, p<$
0.05) (Figure 3). Histology of tumor size examination and apoptosis analysis showed the smallest tumor volume in the combination group, compared with other three groups, which were well correlated with imaging findings (Figure 4).
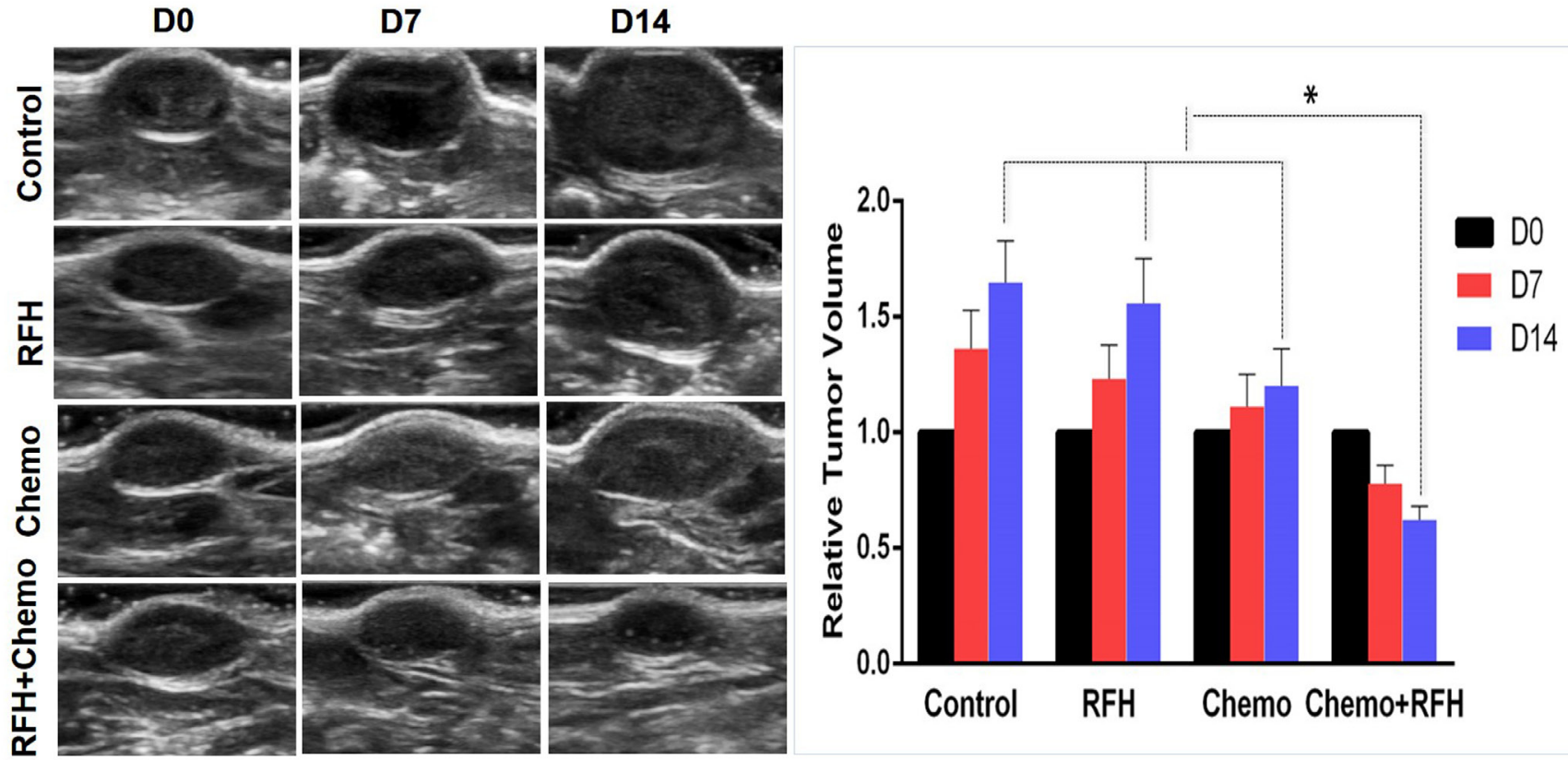

Figure 3: Ultrasound imaging of mice with subcutaneous pancreatic cancers. (Left) Follow tumor growth at days 0,7 and 14 after treatments, showing a significant decrease of average tumor volume in the group with combination therapy (Chemo $+\mathrm{RFH})$, compared to the three control groups (Right, $* p<0.05$ ).

Control
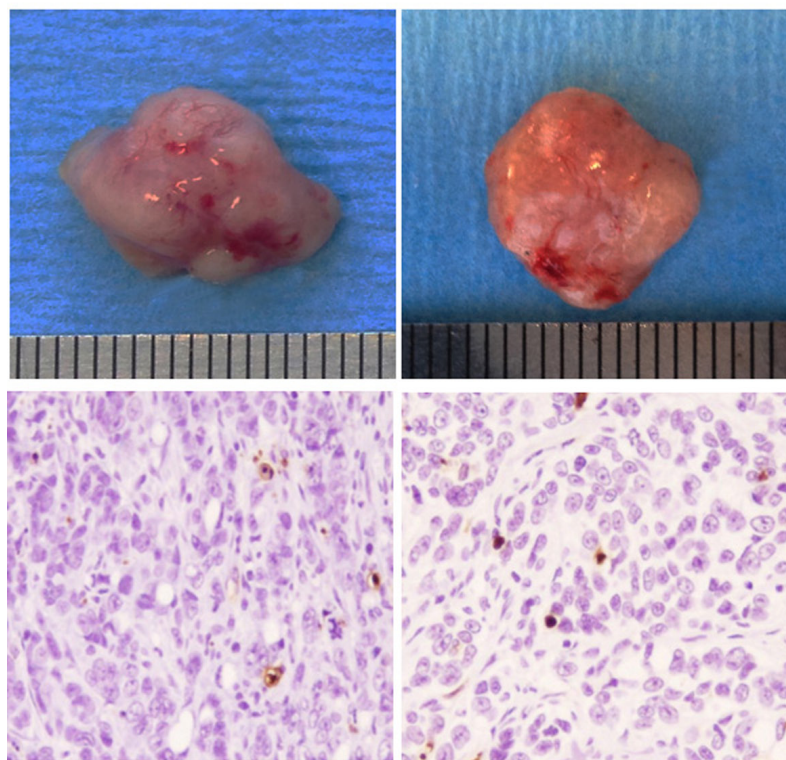

RFH

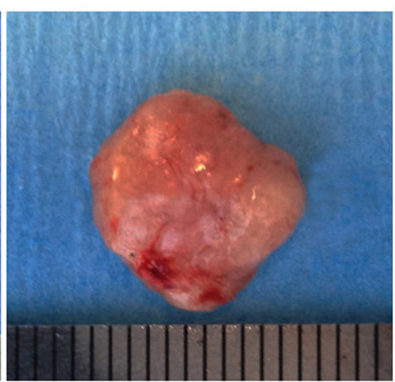

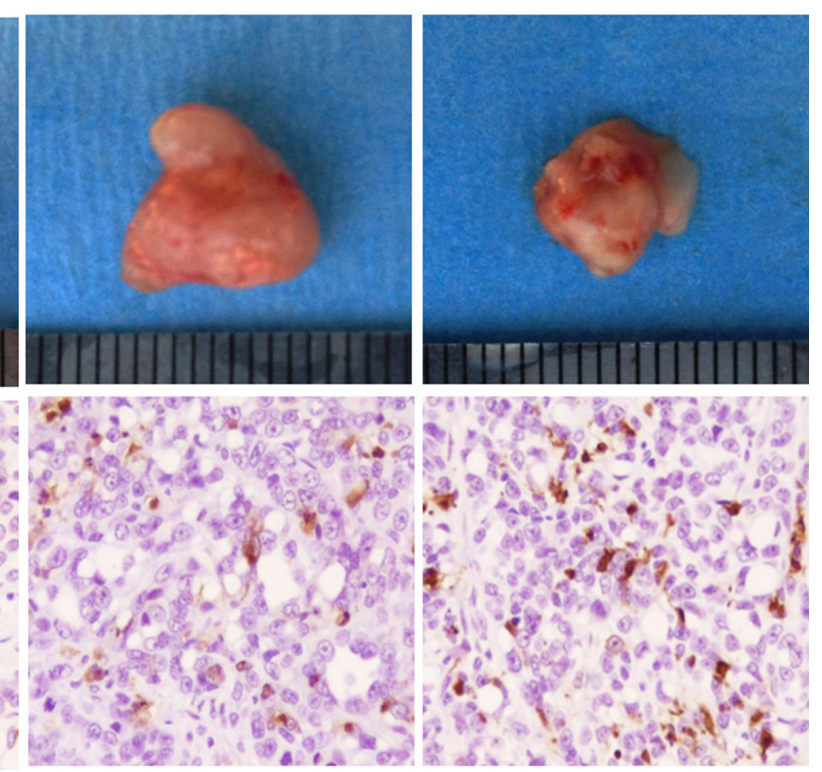

Chemo+RFH

Figure 4: Histology of mice with subcutaneous pancreatic cancers. (Upper row) Representative tumors harvested from four different mouse groups, showing the smallest tumor size in combination therapy group (Chemo+RFH) compared with other three treatments. (Lower row) Apoptosis analysis using TUNEL staining (20X magnification) demonstrates more apoptotic cells (brown dots) in the combination therapy group $(\mathrm{Chemo}+\mathrm{RFH})$ than those of three control groups. 


\section{In vivo validation: $\mathrm{RFH}$-enhanced chemotherapy on rat orthotopic pancreatic cancers}

Chemotherapy plus RFH significantly inhibited the growth of tumors in rat orthotopic pancreatic cancer xenografts (Figure 5). Fourteen days after the treatment, the average relative tumor volume was the smallest in the chemotherapy plus RFH group, as compared with the relative tumor volume of chemotherapy-only group, RFH-only group, and the control group $(0.75 \pm 0.18 \mathrm{VS}$ $1.31 \pm 0.30$ VS $1.61 \pm 0.28$ VS $1.72 \pm 0.28, p<0.05)$. The significant tumor volume decrease in the combination therapy group was well consistent with the results of apoptosis analysis of tumors (Figure 6).

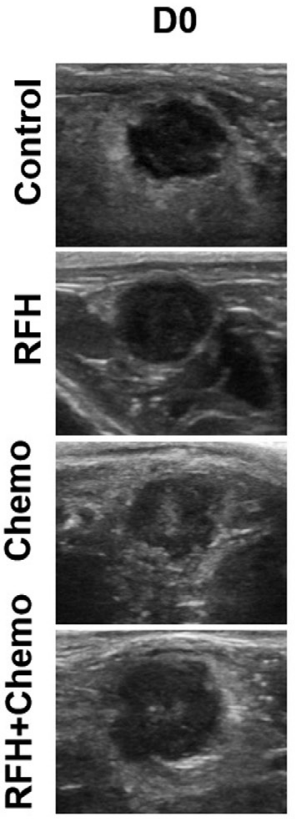

D7

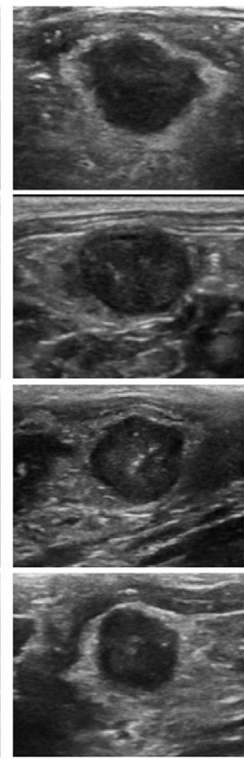

D14

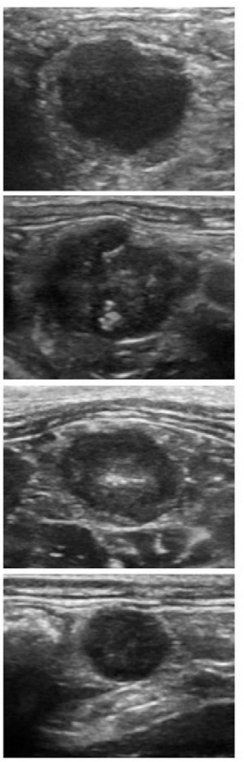

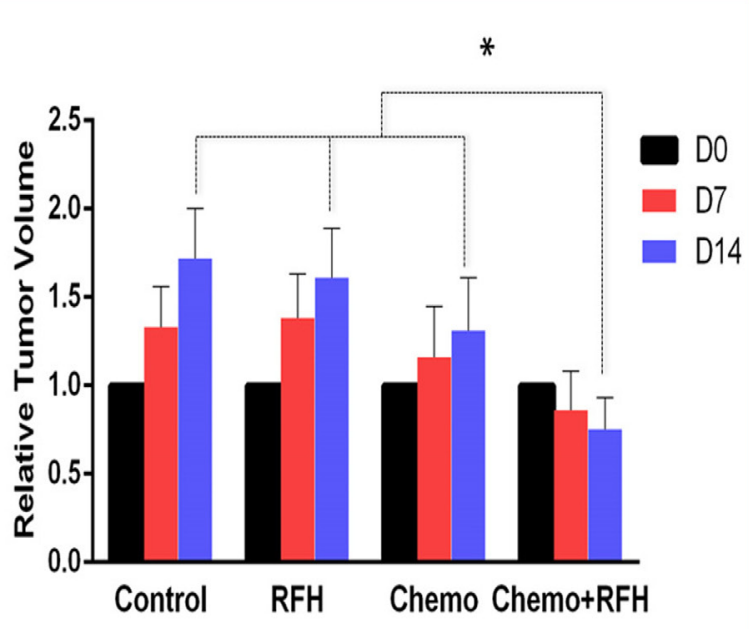

Figure 5: In vivo experiments with rat orthotopic pancreatic cancers. (Left) Representative ultrasound imaging follow-up in four different animal groups with various treatments, showing the smallest tumor size (T) for the combination treatment (RFH+Chemo). (Right) quantified analysis confirmed a significant decreased relative tumor volume in the combination therapy group at day 14 compared with those of other three control animal groups (Right, ${ }^{*} p<0.05$ ).

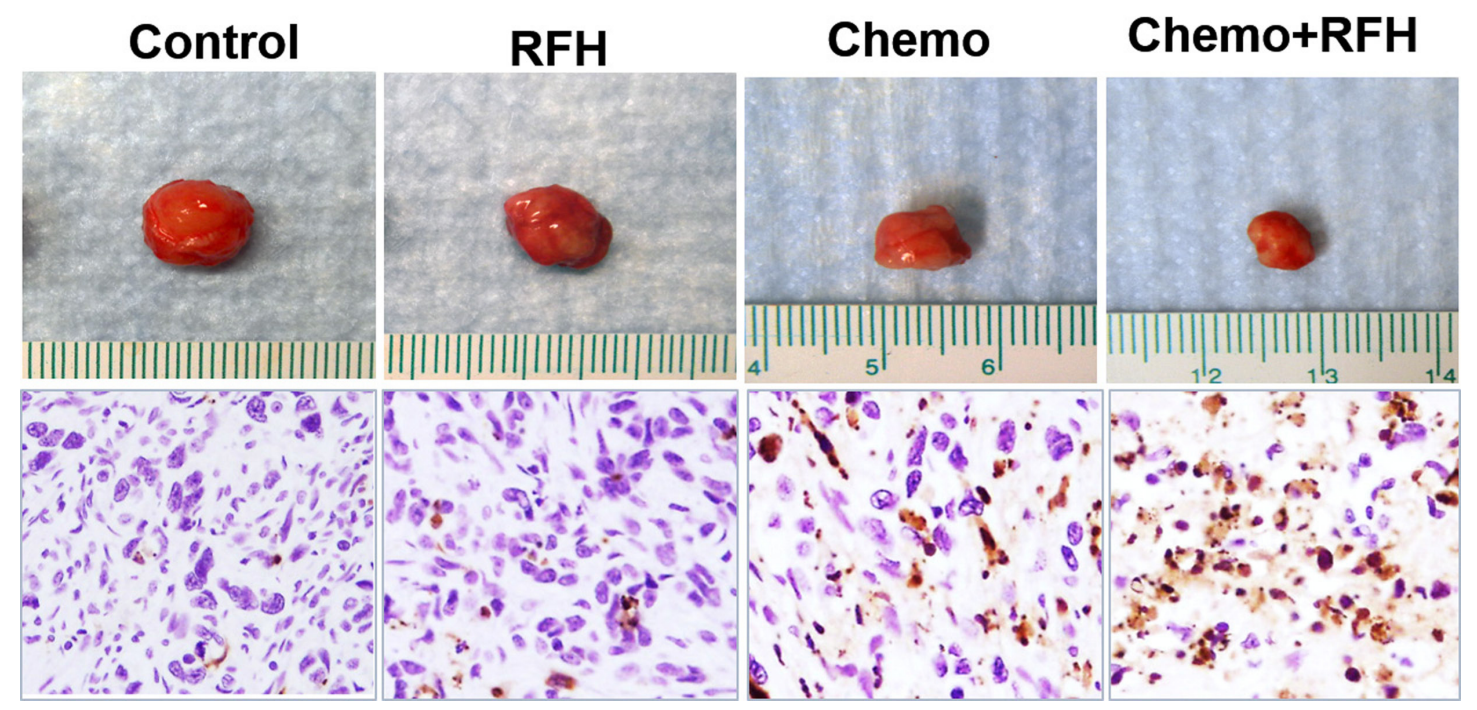

Figure 6: In vivo experiments on rat models with orthotopic pancreatic cancers. (Upper row) Representative tumors harvested from four different animal groups, showing the smallest tumor size in combination therapy group (Chemo+RFH) compared with other three treatments. (Lower row) Apoptosis analysis using TUNEL staining (20X magnification) further confirms more apoptotic cells (brown dots) in the combination therapy group (Chemo+RFH) than those of three control groups. 


\section{DISCUSSION}

In our study, we initially carried out in vitro experiments to establish the "proof-of-principle" of the concept, using RFH to enhance the killing effects of gemcitabine on pancreatic cancer cells. We then further confirmed the principle by in vivo experiments on mouse models with subcutaneous pancreatic cancers. Then, we validated the feasibility of this new technique on rat models with orthotopic pancreatic cancers. We found RFH significantly enhanced the direct intratumoral chemotherapy of pancreatic cancers, both decreasing the number of survived rat pancreatic cancer cells in the invitro experiments and decreasing tumor volumes in both the mouse and rat.

Single-agent gemcitabine is a standard therapeutic strategy for locally advanced and metastatic pancreatic cancer, slightly improving overall survival (OS) and clinical benefit compared with fluorouracil (FU) (5.6 vs. 4.4 months) [21]. However, the overall response rate of pancreatic cancers to gemcitabine remains low $[2,22]$. In current clinical practice, chemotherapy for pancreatic malignancies is usually carried out via systemic administration, limiting the dose of chemotherapeutics to the pancreatic targets and often causing toxicities to other vital organs. Radiofrequency ablation (RFA) is a local ablative method that can destroy tumors by thermal coagulation and protein denaturation. RFA has been used successfully in the treatment of unresectable solid tumors in the liver, lung, and kidney $[3,13]$. The delicate nature of the pancreatic parenchyma predisposes it to pancreatitis caused by RFA-mediated thermal damage. Furthermore, closely adjacent critical structures such as duodenum, portal vein and common bile duct are at risk of thermal injury. These limitations have been the main impeding factors in application of RFA to treat pancreatic cancer [12].

Studies reported that radiofrequency-induced mild hyperthermia can enhance cytotoxicity of many chemotherapeutics through mechanisms of increased drug uptake, increased chemo-sensitivity and decreased the chemoresistance $[1,15,16]$. However, the precise and effective delivery of hyperthermia solely to the target tumor mass remains a serious technological challenge, particularly for deep-seated tumors such as pancreatic malignancies. Under ultrasound imaging guidance, we placed the heating spot of a RF heating guidewire in the center of the pancreatic cancer mass for delivering hyperthermal energy to the tumor. Fiber optical temperature probe placement at the margin of the target tumor allowed precise control RFH within the tumor at a temperature around $42^{\circ} \mathrm{C}$. Our study indicates the potential of taking the advantage of non-ablative hyperthermia to augment the chemotherapy while minimizing RFAassociated thermal compilations.

Development of novel, clinically meaningful therapeutic approaches for pancreatic cancer highly relies on the availability of preclinical animal models that can resemble the anatomic and pathophysiological features of the disease. Such animal models also offer high predictive value for evaluating any new diagnostic and therapeutic technologies [23-25]. Usually, in vivo pancreatic cancer animal models are created on immunodeficient mice [26, 27]. These models suffer from an inherently deficient interaction of implanted cancer cells with host immune system and do not permit evaluation of the treatmentassociated complications to adjacent critical structures[28]. Through the current study, we have successfully created a rat model with orthotopic pancreatic cancer to serve as useful platform for exploring new diagnostic and therapeutic methods.

This study has several limitations. The optimal temperature for maximally enhanced chemotherapeutic effect remains to be characterized and further work in this regard is warranted. This work primarily focused on technical development and suffers from a lack of a systemic chemotherapy control group. We were not able to follow up the animals longer than 14 days, because the tumors in the control groups might grow too large in excess of the institutional-required limit of $10 \%$ body weight.

We concluded that intratumoral RFH can augment the chemotherapeutic effect in an orthotopic pancreatic cancer model. This development may open a new avenue for effective management of pancreatic malignancies by simultaneous integration of radiofrequency technology, interventional oncology, and direct intratumoral chemotherapy.

\section{CONFLICTS OF INTEREST}

Authors have nothing to disclose

\section{GRANT SUPPORT}

This study was supported by an NIH RO1EBO12467 grant.

\section{REFERENCES}

1. Tschoep-Lechner KE, Milani V, Berger F, Dieterle N, Abdel-Rahman S, Salat C and Issels RD. Gemcitabine and cisplatin combined with regional hyperthermia as secondline treatment in patients with gemcitabine-refractory advanced pancreatic cancer. Int J Hyperthermia. 2013; 29(1):8-16.

2. Conroy T, Desseigne F, Ychou M, Bouche O, Guimbaud R, Becouarn Y, Adenis A, Raoul JL, Gourgou-Bourgade S, de la Fouchardiere C, Bennouna J, Bachet JB, KhemissaAkouz F, Pere-Verge D, Delbaldo C, Assenat E, et al. FOLFIRINOX versus gemcitabine for metastatic pancreatic 
cancer. N Engl J Med. 2011; 364(19):1817-1825.

3. Casadei R, Ricci C, Pezzilli R, Serra C, Calculli L, MorselliLabate AM, Santini D and Minni F. A prospective study on radiofrequency ablation locally advanced pancreatic cancer. Hepatobiliary Pancreat Dis Int. 2010; 9(3):306-311.

4. Fegrachi S, Molenaar IQ, Klaessens JH, Besselink MG, Offerhaus JA and van Hillegersberg R. Radiofrequency ablation of the pancreas with and without intraluminal duodenal cooling in a porcine model. J Surg Res. 2013; 184(2):867-872.

5. Fegrachi S, Molenaar IQ, Klaessens JH, Besselink MG, Offerhaus JA and van Hillegersberg R. Radiofrequency ablation of the pancreas: two-week follow-up in a porcine model. Eur J Surg Oncol. 2014; 40(8):1000-1007.

6. Pezzilli R, Serra C, Ricci C, Casadei R, Monari F, D’Ambra $\mathrm{M}$ and Minni F. Radiofrequency ablation for advanced ductal pancreatic carcinoma: is this approach beneficial for our patients? A systematic review. Pancreas. 2011; 40(1):163-165.

7. Rossi M, Orgera G, Hatzidakis A and Krokidis M. Minimally invasive ablation treatment for locally advanced pancreatic adenocarcinoma. Cardiovasc Intervent Radiol. 2014; 37(3):586-591.

8. Gavini $\mathrm{H}$ and Lee JH. Endoscopic ultrasound-guided endotherapy. J Clin Gastroenterol. 2015; 49(3):185-193.

9. Date RS, Biggins J, Paterson I, Denton J, McMahon RF and Siriwardena AK. Development and validation of an experimental model for the assessment of radiofrequency ablation of pancreatic parenchyma. Pancreas. 2005; 30(3):266-271.

10. Gaidhane M, Smith I, Ellen K, Gatesman J, Habib N, Foley P, Moskaluk C and Kahaleh M. Endoscopic UltrasoundGuided Radiofrequency Ablation (EUS-RFA) of the Pancreas in a Porcine Model. Gastroenterol Res Pract. 2012; 2012:431451.

11. Rostas JW, Richards WO and Thompson LW. Improved rate of pancreatic fistula after distal pancreatectomy: parenchymal division with the use of saline-coupled radiofrequency ablation. HPB (Oxford). 2012; 14(8):560564.

12. Date RS. Current status of local ablative techniques in the treatment of pancreatic cancer. Pancreas. 2006; 33(2):198199.

13. Girelli R, Frigerio I, Salvia R, Barbi E, Tinazzi Martini $\mathrm{P}$ and Bassi C. Feasibility and safety of radiofrequency ablation for locally advanced pancreatic cancer. Br J Surg. 2010; 97(2):220-225.

14. Carrafiello G, Ierardi AM, Fontana F, Petrillo M, Floridi C, Lucchina N, Cuffari S, Dionigi G, Rotondo A and Fugazzola C. Microwave ablation of pancreatic head cancer: safety and efficacy. J Vasc Interv Radiol. 2013; 24(10):1513-1520.

15. Zhang $\mathrm{T}$, Zhang F, Meng $\mathrm{Y}$, Wang $\mathrm{H}$, Le $\mathrm{T}$, Wei B, Lee D, Willis P, Shen B and Yang X. Diffusion-weighted MRI monitoring of pancreatic cancer response to radiofrequency heat-enhanced intratumor chemotherapy. NMR in biomedicine. 2013; 26(12):1762-1767.

16. Zhang F, Le T, Wu X, Wang H, Zhang T, Meng Y, Wei $\mathrm{B}$, Soriano SS, Willis $\mathrm{P}$, Kolokythas $\mathrm{O}$ and Yang $\mathrm{X}$. Intrabiliary RF heat-enhanced local chemotherapy of a cholangiocarcinoma cell line: monitoring with dualmodality imaging--preclinical study. Radiology. 2014; 270(2):400-408.

17. Ishikawa $\mathrm{T}$, Kokura $\mathrm{S}$, Sakamoto $\mathrm{N}$, Ando $\mathrm{T}$, Imamoto E, Hattori T, Oyamada H, Yoshinami N, Sakamoto M, Kitagawa K, Okumura Y, Yoshida N, Kamada K, Katada K, Uchiyama K, Handa O, et al. Phase II trial of combined regional hyperthermia and gemcitabine for locally advanced or metastatic pancreatic cancer. International journal of hyperthermia. 2012; 28(7):597-604.

18. Bakshandeh-Bath A, Stoltz AS, Homann N, Wagner T, Stolting S and Peters SO. Preclinical and clinical aspects of carboplatin and gemcitabine combined with whole-body hyperthermia for pancreatic adenocarcinoma. Anticancer research. 2009; 29(8):3069-3077.

19. Yang X. Imaging of vascular gene therapy. Radiology. 2003; 228(1):36-49.

20. Du X, Qiu B, Zhan X, Kolmakova A, Gao F, Hofmann LV, Cheng L, Chatterjee S and Yang X. Radiofrequencyenhanced vascular gene transduction and expression for intravascular MR imaging-guided therapy: feasibility study in pigs. Radiology. 2005; 236(3):939-944.

21. DiMaio CJ, DeWitt JM and Brugge WR. Ablation of pancreatic cystic lesions: the use of multiple endoscopic ultrasound-guided ethanol lavage sessions. Pancreas. 2011; 40(5):664-668.

22. Herrmann R, Bodoky G, Ruhstaller T, Glimelius B, Bajetta E, Schuller J, Saletti P, Bauer J, Figer A, Pestalozzi B, Kohne CH, Mingrone W, Stemmer SM, Tamas K, Kornek GV, Koeberle D, et al. Gemcitabine plus capecitabine compared with gemcitabine alone in advanced pancreatic cancer: a randomized, multicenter, phase III trial of the Swiss Group for Clinical Cancer Research and the Central European Cooperative Oncology Group. J Clin Oncol. 2007; 25(16):2212-2217.

23. Yamaguchi T, Ikehara S, Nakanishi H and Ikehara Y. A genetically engineered mouse model developing rapid progressive pancreatic ductal adenocarcinoma. J Pathol. 2014; 234(2):228-238.

24. Mazur PK and Siveke JT. Genetically engineered mouse models of pancreatic cancer: unravelling tumour biology and progressing translational oncology. Gut. 2012; 61(10):1488-1500.

25. Singh M, Murriel CL and Johnson L. Genetically engineered mouse models: closing the gap between preclinical data and trial outcomes. Cancer Res. 2012; 72(11):2695-2700.

26. Perez-Mancera PA, Guerra C, Barbacid M and Tuveson DA. What we have learned about pancreatic cancer from 
mouse models. Gastroenterology. 2012; 142(5):1079-1092.

27. Mazur PK, Herner A, Neff F and Siveke JT. Current methods in mouse models of pancreatic cancer. Methods Mol Biol. 2015; 1267:185-215.

28. Partecke IL, Kaeding A, Sendler M, Albers N, Kuhn JP, Speerforck S, Roese S, Seubert F, Diedrich S, Kuehn S, Weiss UF, Mayerle J, Lerch MM, Hadlich S, Hosten N, Heidecke $\mathrm{CD}$, et al. In vivo imaging of pancreatic tumours and liver metastases using 7 Tesla MRI in a murine orthotopic pancreatic cancer model and a liver metastases model. BMC Cancer. 2011; 11:40. 